RASĀYAN J. Chem.

47-63| Special Issue | 2021

ISSN: 0974-1496 | e-ISSN: 0976-0083 | CODEN: RJCABP

http://www.rasayanjournal.com

http://www.rasayanjournal.co.in

\title{
A CSD ANALYSIS OF SOME CHLORINATED QUINOLINES
}

\author{
J. Stondus ${ }^{1}$, S. Anthal ${ }^{2}$ and R. Kant ${ }^{1, \bowtie}$ \\ ${ }^{1} \mathrm{X}$-ray Crystallography Laboratory, Department of Physics, University of Jammu \\ Jammu, 180006 (J\&K) India \\ ${ }^{2}$ Government Degree College (Boys) Udhampur, 182101, J\&K, India \\ ${ }^{\otimes}$ Corresponding Author: rkant.ju@gmail.com
}

Quinoline and its derivatives, especially the halogenated quinolines, possess a variety of biological activities and exhibit some excellent antimicrobial and anti-cancer activities. The chemical and crystallographic aspects of this class of chemical compounds have been studied in isolation over the years, but there seems to be no attempt in the literature that could present and place together with the comparative crystal structures and their biological aspects for a larger audience comprising of small molecule crystallographers, chemists, biophysicists, etc. In order to fill the gap, a CSD survey of the X-ray structures of some interesting and similar-looking chlorine quinoline derivatives was made for obtaining the comparative crystal data, empirical analysis of the predicted biological activities, and molecular packing interaction analysis. The intermolecular interactions $[X-H . . . A(X=C, N, O ; A=O, C L, N, C, F)]$ and intra-molecular interactions $[\mathrm{X}-\mathrm{H} \ldots \mathrm{A}(\mathrm{X}=\mathrm{C}, \mathrm{N} ; \mathrm{A}=\mathrm{CL}, \mathrm{O}, \mathrm{C}, \mathrm{F}, \mathrm{N})]$ have been computed and analyzed for a better understanding of the molecular packing in halogenated quinolines.

Keywords: Halogenated, Quinoline, Biological Activity, Comparative Analysis, Hydrogen Bonding.

RASĀYANJ. Chem., Special Issue, 2021

\section{INTRODUCTION}

Quinoline $\left(\mathrm{C}_{9} \mathrm{H}_{7} \mathrm{~N}\right)$ is a heterocyclic aromatic compound (Fig.-1) that is found in natural compounds and biologically active substances, exhibiting a wide range of activity. Many activities of quinoline derivatives such as antimicrobial activity, ${ }^{1}$ antimycobacterial activity, ${ }^{2}$ inhibitors for plaque-forming microorganisms, ${ }^{3}$ anti-cancer activity, ${ }^{4}$ antitubercular and antiamoebic activities, ${ }^{5}$ antileishmanial activity ${ }^{6}$ have been reported in the literature. A careful CSD survey (version 2.0.5, 2021) of a few structurallysimilar crystal structures of quinoline derivatives revealed that halogenated quinoline derivatives with amino or nitrogen groups present in the ring system exhibits excellent cytotoxic activities and are useful as antimicrobial and anticancer agents. ${ }^{7,8}$ In view of some wide-ranging properties of halogenated quinoline derivatives, we have identified a series of chemically-similar-looking chlorine substituted quinoline derivatives for a close comparative analysis of their crystallographic structures and inter/intramolecular hydrogen bond interactions. A total of twenty-six such crystal structures have been identified for the analysis and their CCDC codes, IUPAC names/ chemical structures, chemical formulas, and corresponding published references are presented in Table-1.9-28

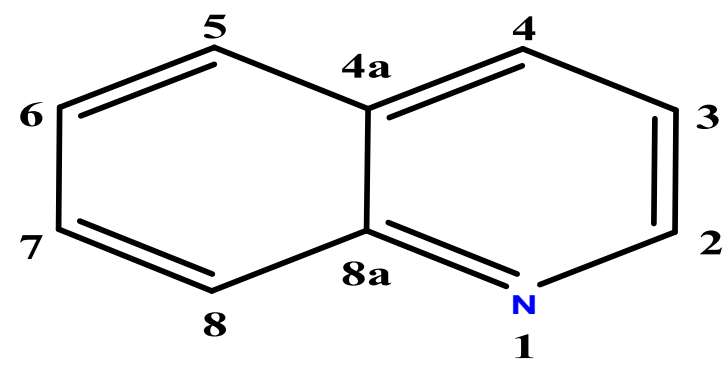

Fig.-1: Basic Quinoline Structure with Atomic Labeling 
RASĀYAN J. Chem.

47-63| Special Issue | 2021

Table-1: CCDC Codes, Chemical Structures / IUPAC Names, Molecular Formula and References of the Selected Compounds

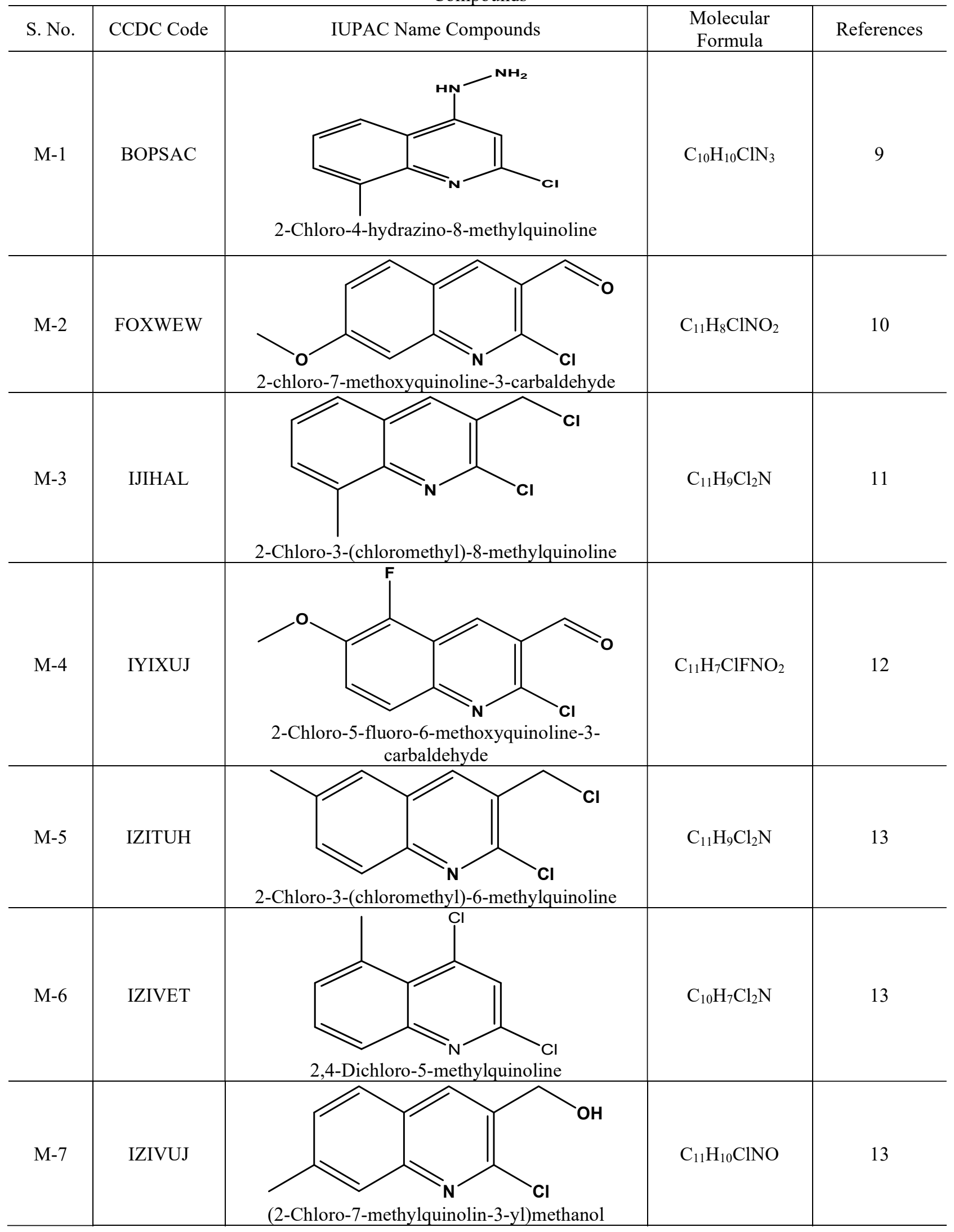


RASĀYAN J. Chem.

47-63| Special Issue | 2021

\begin{tabular}{|c|c|c|c|c|}
\hline M-8 & PINVEN & 2-Chloro-4-chloromethyl-6,7-dimethylquinoline & $\mathrm{C}_{12} \mathrm{H}_{11} \mathrm{Cl}_{2} \mathrm{~N}$ & 14 \\
\hline M-9 & PUHFOO & 2-Chloro-7-methylquinoline-3-carbaldehyde & $\mathrm{C}_{11} \mathrm{H}_{8} \mathrm{ClNO}$ & 15 \\
\hline M-10 & PUHFUU & 2-Chloro-6-methoxyquinoline-3-carbaldehyde & $\mathrm{C}_{11} \mathrm{H}_{8} \mathrm{ClNO}_{2}$ & 16 \\
\hline M-11 & PUHGAB & 2-Chloroquinoline-3-carbaldehyde & $\mathrm{C}_{10} \mathrm{H}_{6} \mathrm{ClNO}$ & 17 \\
\hline M-12 & PUHGEF & 2-Chloro-8-methoxyquinoline-3-carbaldehyde & $\mathrm{C}_{11} \mathrm{H}_{8} \mathrm{ClNO}_{2}$ & 18 \\
\hline M-13 & PUHGIJ & 2-Chloro-7,8-dimethylquinoline-3-carbaldehyde & $\mathrm{C}_{12} \mathrm{H}_{10} \mathrm{ClNO}$ & 19 \\
\hline M-14 & PUHHIK & 2-Chloro-6-methylquinoline-3-carbaldehyde & $\mathrm{C}_{11} \mathrm{H}_{8} \mathrm{ClNO}$ & 20 \\
\hline M-15 & PUKJUB & 2-Chloroquinoline-3-carboxylic acid & $\mathrm{C}_{10} \mathrm{H}_{6} \mathrm{ClNO}_{2}$ & 21 \\
\hline
\end{tabular}


RASĀYAN J. Chem.

47-63| Special Issue | 2021

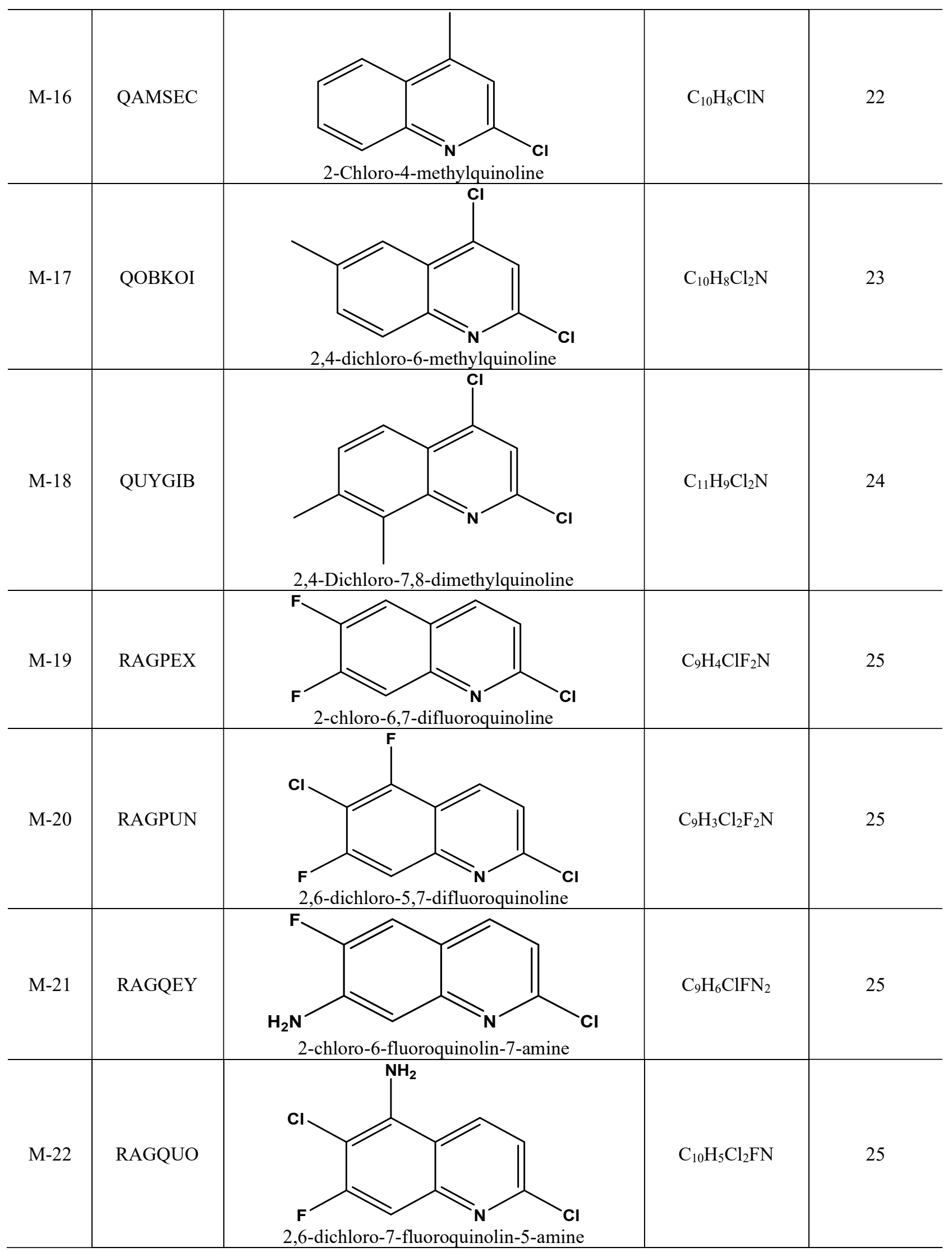


RASĀYAN J. Chem.

47-63| Special Issue | 2021

(2-23

\section{Crystallographic Analysis}

A preliminary crystal data of the structures, as given in Table-2, reveals that most of the structures have preferred to crystallize in monoclinic crystal system $(\sim 65 \%)$, followed by orthorhombic $(\sim 27 \%)$ and triclinic $(\sim 3 \%)$, and this observation is in agreement with the findings of Stout and Jensen. ${ }^{29}$ The incidence of occurrence of the space group $\mathrm{P} 2{ }_{1} / \mathrm{n}$ (or P $2_{1} / \mathrm{c}$ ) is also quite high $(58 \%)$ and the reliability index for all the structures (i.e. $\mathrm{R}_{\text {range }}=0.0202-0.0852$ ) indicates that all these structures were obtained at a good to moderate level of refinement.

Table-2: The Crystal Systems, Space Groups, Z-Values, Study Temperatures and R-Factors of the Molecules M-1 to

\begin{tabular}{c|c|c|c|c|c}
\hline S. No. & Crystal System & $\begin{array}{c}\text { Space Group } \\
\text { Symbol }\end{array}$ & Z Value & Study Temp. (K) & R-factor (\%) \\
\hline M-1 & Monoclinic & $\mathrm{P}_{1} / n$ & 8 & 100 & 4.85 \\
\hline M-2 & Monoclinic & $\mathrm{P} 2_{1} / n$ & 4 & 296 & 6.20 \\
\hline M-3 & Monoclinic & $\mathrm{P}_{1} / c$ & 4 & 100 & 2.02 \\
\hline M-4 & Monoclinic & $\mathrm{P} 2_{1} / c$ & 4 & 120 & 3.81 \\
\hline M-5 & Triclinic & $\mathrm{P}-1$ & 2 & 295 & 3.46 \\
\hline M-6 & Monoclinic & $\mathrm{P} 2_{1} / c$ & 4 & 295 & 3.28 \\
\hline
\end{tabular}


RASĀYAN J. Chem.

47-63| Special Issue | 2021

\begin{tabular}{|c|c|c|c|c|c|}
\hline M-7 & Monoclinic & $\mathrm{P} 2{ }_{1} / c$ & 8 & 290 & 4.27 \\
\hline M-8 & Orthorhombic & $\mathrm{Pca}_{1}$ & 4 & 293 & 3.50 \\
\hline M-9 & Monoclinic & $\mathrm{P} 2_{1} / n$ & 4 & 290 & 7.79 \\
\hline M-10 & Monoclinic & $\mathrm{P} 2{ }_{1} / c$ & 4 & 290 & 3.81 \\
\hline M-11 & Monoclinic & $\mathrm{P} 2_{1} / n$ & 4 & 290 & 3.30 \\
\hline M-12 & Monoclinic & $\mathrm{P} 2{ }_{1} / n$ & 4 & 290 & 3.66 \\
\hline M-13 & Orthorhombic & Pnma & 4 & 290 & 3.41 \\
\hline M-14 & Monoclinic & Pc & 2 & 290 & 3.40 \\
\hline M-15 & Orthorhombic & $\mathrm{P} 2{ }_{1} n b$ & 4 & 120 & 3.76 \\
\hline M-16 & Orthorhombic & $\mathrm{P} b c a$ & 8 & 150 & 4.3 \\
\hline M-17 & Triclinic & P-1 & 4 & 293 & 5.77 \\
\hline M-18 & Orthorhombic & $\mathrm{Pca} 2_{1}$ & 8 & 295 & 4.90 \\
\hline M-19 & Monoclinic & $\mathrm{C} 2 / c$ & 8 & 200 & 2.92 \\
\hline $\mathrm{M}-20$ & Monoclinic & $\mathrm{P} 2{ }_{1} / c$ & 4 & 296 & 6.91 \\
\hline M-21 & Orthorhombic & $\mathrm{P} 2{ }_{1} 2_{1} 2_{1}$ & 4 & 296 & 3.24 \\
\hline M-22 & Monoclinic & $\mathrm{P} 2{ }_{1} / c$ & 4 & 296 & 2.96 \\
\hline M-23 & Orthorhombic & $\mathrm{P} 2{ }_{1} 2_{1} 2_{1}$ & 4 & 296 & 2.73 \\
\hline M-24 & Monoclinic & $\mathrm{P} 2{ }_{1} / n$ & 4 & 296 & 4.09 \\
\hline M-25 & Monoclinic & $\mathrm{P} 2{ }_{1} / c$ & 4 & 290 & 6.06 \\
\hline M-26 & Monoclinic & $\mathrm{P} 2{ }_{1} / c$ & 4 & 295 & 8.52 \\
\hline
\end{tabular}

The precise data in terms of various bond distances between C-N $\left(\mathrm{sp}^{3}-\mathrm{sp}^{2} / \mathrm{sp}^{2}-\mathrm{sp}^{3}\right), \mathrm{C}-\mathrm{C}\left(\mathrm{sp}^{3}-\mathrm{sp}^{2} / \mathrm{sp}^{2}-\right.$ $\left.\mathrm{sp}^{3}\right), \mathrm{C}-\mathrm{Cl}\left(\mathrm{sp}^{2}-\mathrm{sp}^{3}\right)$, are presented in Table-3. The $\mathrm{C} 2-\mathrm{N} 1$ bond distance, ranging between $1.274-1.308 \AA$ (average, $1.296 \AA$ ) is much shorter than the standard value of $1.336 \AA .{ }^{30}$ In all the structures of the series, this bond distance is shorter compared to the corresponding value $(1.319 \AA)$ of the simple quinoline structure. ${ }^{31}$ Similarly, the bond distance N1-C8a $\left(\mathrm{sp}^{3}-\mathrm{sp}^{2} / \mathrm{sp}^{2}-\mathrm{sp}^{3}\right)$ lies in the range of $1.361-1.389 \AA$ (average value $1.372 \AA$ ), the average value bond distance in all structures is in agreement with the standard literature value $(1.374 \AA) .{ }^{31}$ The bond distance C2-C3 $\left(\mathrm{sp}^{3}-\mathrm{sp}^{2} / \mathrm{sp}^{2}-\mathrm{sp}^{3}\right)$, lies in the range $1.389-$ $1.433 \AA$ (average being $1.413 \AA$ ). This bond distance in most of the structures, except M-1 (1.389 $\AA$ ), M-6

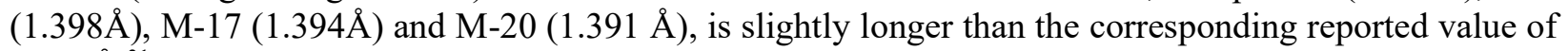
$1.400 \AA .^{31}$

Table-3: Selected Bond Distances and Bond Angles around C2 of the Molecules M-1 to M-26.

\begin{tabular}{c|c|c|c|c|c|c|c}
\hline \multirow{2}{*}{ S.No. } & \multicolumn{3}{|c|}{ Selected Bond Distances $(\AA)$} & \multicolumn{2}{c}{ Selected Bond Angles Around C2 $\left(^{\circ}\right)$} \\
\cline { 2 - 8 } & $\begin{array}{c}\mathrm{N} 1-\mathrm{C} 2 \\
\left(\mathrm{sp}^{3}-\mathrm{sp}^{2}\right. \\
\left.\mathrm{Sp}^{2}-\mathrm{sp}^{3}\right)\end{array}$ & $\begin{array}{c}\mathrm{N} 1-\mathrm{C} 8 \mathrm{a} \\
\left(\mathrm{sp}^{3}-\mathrm{sp}^{2}\right. \\
\left.\mathrm{Sp}^{2}-\mathrm{sp}^{3}\right)\end{array}$ & $\begin{array}{c}\mathrm{C} 2-\mathrm{C} 3 \\
\left(\mathrm{sp}^{3}-\mathrm{sp}^{2}\right. \\
\left.\mathrm{Sp}^{2}-\mathrm{sp}^{3}\right)\end{array}$ & $\begin{array}{c}\mathrm{C} 2-\mathrm{Cl} \\
\left(\mathrm{sp}^{2}-\mathrm{sp}^{3}\right)\end{array}$ & N1-C2-C3 & N1-C2-Cl & C3-C2-Cl \\
\hline $\mathrm{M}-1$ & 1.308 & 1.377 & 1.389 & 1.755 & 128.60 & 115.22 & 116.53 \\
\hline $\mathrm{M}-2$ & 1.292 & 1.376 & 1.410 & 1.749 & 126.21 & 115.00 & 118.79 \\
\hline $\mathrm{M}-3$ & 1.303 & 1.372 & 1.425 & 1.742 & 126.04 & 116.19 & 117.77 \\
\hline $\mathrm{M}-4$ & 1.297 & 1.375 & 1.422 & 1.754 & 125.78 & 115.06 & 119.15 \\
\hline $\mathrm{M}-5$ & 1.295 & 1.371 & 1.419 & 1.750 & 126.48 & 115.44 & 118.06 \\
\hline $\mathrm{M}-6$ & 1.289 & 1.371 & 1.398 & 1.745 & 125.48 & 117.09 & 117.43 \\
\hline $\mathrm{M}-7$ & 1.308 & 1.373 & 1.413 & 1.747 & 127.22 & 115.12 & 117.81 \\
\hline
\end{tabular}


RASĀYAN J. Chem.

47-63| Special Issue | 2021

\begin{tabular}{c|c|c|c|c|c|c|c}
\hline $\mathrm{M}-8$ & 1.290 & 1.367 & 1.425 & 1.746 & 126.59 & 116.76 & 116.62 \\
\hline $\mathrm{M}-9$ & 1.293 & 1.374 & 1.422 & 1.753 & 125.71 & 115.70 & 118.59 \\
\hline $\mathrm{M}-10$ & 1.302 & 1.362 & 1.414 & 1.746 & 125.38 & 114.07 & 119.62 \\
\hline $\mathrm{M}-11$ & 1.288 & 1.372 & 1.423 & 1.752 & 126.16 & 115.13 & 118.71 \\
\hline $\mathrm{M}-12$ & 1.294 & 1.365 & 1.423 & 1.754 & 125.95 & 114.99 & 119.05 \\
\hline $\mathrm{M}-13$ & 1.290 & 1.378 & 1.419 & 1.754 & 126.60 & 115.19 & 118.21 \\
\hline $\mathrm{M}-14$ & 1.299 & 1.366 & 1.409 & 1.748 & 126.42 & 114.65 & 118.93 \\
\hline $\mathrm{M}-15$ & 1.306 & 1.389 & 1.424 & 1.736 & 124.91 & 113.58 & 121.52 \\
\hline $\mathrm{M}-16$ & 1.293 & 1.375 & 1.410 & 1.750 & 126.21 & 116.55 & 117.23 \\
\hline $\mathrm{M}-17$ & 1.297 & 1.361 & 1.394 & 1.737 & 125.98 & 116.47 & 116.73 \\
\hline $\mathrm{M}-18$ & 1.274 & 1.383 & 1.421 & 1.720 & 125.58 & 115.92 & 115.78 \\
\hline $\mathrm{M}-19$ & 1.306 & 1.372 & 1.412 & 1.742 & 126.38 & 116.74 & 116.88 \\
\hline $\mathrm{M}-20$ & 1.307 & 1.372 & 1.391 & 1.745 & 126.46 & 115.84 & 117.69 \\
\hline $\mathrm{M}-21$ & 1.299 & 1.376 & 1.403 & 1.742 & 126.24 & 116.15 & 117.61 \\
\hline $\mathrm{M}-22$ & 1.297 & 1.368 & 1.403 & 1.743 & 125.83 & 116.19 & 117.98 \\
\hline $\mathrm{M}-23$ & 1.295 & 1.361 & 1.407 & 1.746 & 125.57 & 115.75 & 118.68 \\
\hline $\mathrm{M}-24$ & 1.300 & 1.374 & 1.410 & 1.745 & 126.50 & 116.79 & 116.77 \\
\hline M-25 & 1.307 & 1.373 & 1.419 & 1.735 & 125.97 & 116.18 & 117.85 \\
\hline M-26 & 1.276 & 1.376 & 1.433 & 1.751 & 127.95 & 115.88 & 116.14 \\
\hline Largest & 1.308 & 1.389 & 1.433 & 1.755 & 128.6 & 117.09 & 121.52 \\
\hline Smallest & 1.274 & 1.361 & 1.389 & 1.720 & 124.91 & 113.58 & 115.78 \\
\hline Average & 1.296 & 1.372 & 1.413 & 1.746 & 126.24 & 115.68 & 117.93 \\
\hline
\end{tabular}

The shortening and lengthening of bond distances N1-C2 and $\mathrm{C} 2-\mathrm{C} 3$ could possibly be due to the presence of a highly electronegative chlorine atom located at $\mathrm{C} 2$ position and its involvement in the formation of hydrogen bond interactions. The carbon-chlorine bond distance [C2- $\left.\mathrm{Cl}\left(\mathrm{sp}^{2}-\mathrm{sp}^{3}\right)\right]$, lying in the range of $1.720-1.775 \AA$ (average being $1.746 \AA$ ),shows a slight departure from the standard value $1.739 \AA .{ }^{30}$ The bond angle N1-C2-C3 lies in the range of $124.91^{\circ}-128.60^{\circ}$. The average value $\left(126.19^{\circ}\right)$ for this bond angle in the basic quinoline structure is $124.49^{\circ}$, indicating a slight but not very significant deviation. The other bond angles of interest, viz. N1-C2-Cl and $\mathrm{C} 3-\mathrm{C} 2-\mathrm{Cl}$, lie in the range $113.58^{\circ}-$ $118.11^{\circ}\left(\right.$ average $\left.=115.76^{\circ}\right)$ and $115.78^{\circ}-121.52^{\circ}\left(\right.$ average $\left.=117.93^{\circ}\right)$, respectively.

\section{Biological Activity Prediction}

Activities are the intrinsic properties of chemical compounds and the activity spectrum forms the basis for the prediction of biological activities, which depend only on their chemical structures. The Prediction of Activity Spectra for chemical Substances (PASS software ${ }^{32}$ ), a large database housing chemical structures of a huge number of organic compounds as described by the original descriptor called Multilevel Neighbourhoods of Atoms (MNA), ${ }^{33}$ has been employed to show that the MNA descriptors are capable of representing various structure-property relationships, including many types of biological activity ${ }^{34}$ and drug-likeness, ${ }^{35}$ with the structural formula of the compound in .mol file as the input and the output represents a list of probable activities, i.e., $\mathrm{Pa}$ (probability of being active) and $\mathrm{Pi}$ (probability of being inactive), respectively, on a scale of $0-1$. Though most of the structures have shown enormous biological activities, yet we have retained those with $\mathrm{Pa} \geq 0.5$ (Table-4).

Table-4: Comparison Table of Some Biological Activities as predicted by PASS Online Software

\begin{tabular}{c|c|c|c|c|c}
\hline S.No. & $\begin{array}{c}\text { Anti- } \\
\text { inflammatory }\end{array}$ & $\begin{array}{c}\text { Membrane } \\
\text { permeability } \\
\text { Inhibitor }\end{array}$ & $\begin{array}{c}\text { Antiviral } \\
\text { (Rhinovirus) }\end{array}$ & $\begin{array}{c}\text { Complement Factor } \\
\text { D inhibitor }\end{array}$ & $\begin{array}{c}\text { Phobic Disorders } \\
\text { Treatment }\end{array}$ \\
\hline M-1 & - & - & - & $0.455>0.092$ & - \\
\hline M-2 & - & $0.321>0.238$ & $0.394>0.097$ & $0.557>0.050$ & $0.414>0.224$ \\
\hline M-3 & $0.605>0.061$ & - & $0.369>0.131$ & $0.563>0.049$ & $0.533>0.156$ \\
\hline M-4 & - & - & $0.306>0.241$ & $0.375>0.144$ & - \\
\hline M-5 & - & $0.281>0.255$ & $0.386>0.108$ & $0.517>0.064$ & $0.497>0.175$ \\
\hline
\end{tabular}


RASĀYAN J. Chem.

47-63| Special Issue | 2021

\begin{tabular}{c|c|c|c|c|c}
\hline M-6 & $0.538>0.046$ & $0.523>0.134$ & $0.411>0.079$ & $0.666>0.024$ & $0.697>0.077$ \\
\hline M-7 & $0.314>0.148$ & $0.465>0.169$ & $0.427>0.065$ & - & $0.536>0.154$ \\
\hline M-8 & - & - & - & $0.484>0.078$ & $0.382>0.249$ \\
\hline M-9 & - & $0.447>0.179$ & $0.389>0.104$ & $0.532>0.059$ & $0.492>0.178$ \\
\hline M-10 & $0.793>0.006$ & $0.303>0.245$ & - & $0.572>0.046$ & $0.370>0.259$ \\
\hline M-11 & $0.235>0.135$ & $0.342>0.229$ & $0.358>0.146$ & $0.661>0.025$ & $0.638>0.105$ \\
\hline M-12 & $0.248>0.215$ & - & $0.378>0.118$ & $0.543>0.055$ & $0.455>0.200$ \\
\hline M-13 & $0.287>0.174$ & - & $0.369>0.140$ & $0.532>0.059$ & $0.492>0.178$ \\
\hline M-14 & - & $0.396>0.205$ & $0.390>0.103$ & $0.524>0.061$ & $0.499>0.174$ \\
\hline M-15 & $0.405>0.093$ & $0.549>0.118$ & $0.485>0.030$ & $0.670>0.024$ & $0.709>0.072$ \\
\hline M-16 & - & $0.495>0.152$ & $0.429>0.063$ & $0.696>0.019$ & $0.676>0.087$ \\
\hline M-17 & - & $0.547>0.119$ & $0.426>0.066$ & $0.628>0.032$ & $0.665>0.092$ \\
\hline M-18 & $0.582>0.036$ & $0.474>0.164$ & $0.417>0.073$ & $0.669>0.024$ & $0.701>0.075$ \\
\hline M-19 & - & $0.369>0.218$ & $0.337>0.181$ & $0.616>0.034$ & $0.658>0.096$ \\
\hline M-20 & - & $0.287>0.252$ & $0.328>0.198$ & $0.626>0.032$ & $0.670>0.090$ \\
\hline M-21 & $0.241>0.200$ & - & - & $0.520>0.063$ & $0.577>0.144$ \\
\hline M-22 & - & $0.370>0.217$ & - & $0.563>0.049$ & $0.623>0.112$ \\
\hline M-23 & $0.217>0.163$ & - & - & $0.520>0.063$ & $0.557>0.144$ \\
\hline M-24 & $0.517>0.052$ & $0.568>0.106$ & $0.423>0.068$ & $0.781>0.008$ & $0.791>0.037$ \\
\hline M-25 & $0.318>0.145$ & - & $0.414>0.076$ & $0.432>0.105$ & $0.578>0.134$ \\
\hline M-26 & $0.294>0.166$ & $0.417>0.195$ & $0.428>0.064$ & - & $0.543>0.151$ \\
\hline & & & & & \\
\hline
\end{tabular}

The antiviral activity has extensively been demonstrated by most of the molecules with $\mathrm{Pa}>\mathrm{Pi}$. The antiinflammatory activity has been found in molecules M-3, M-6, M-10, M-18 and M-24, while the moleculesM-6, M-15, M-17 and M-24 indicate their relevance as membrane permeability inhibitors.

\section{Hydrogen Bonding Interactions Analysis}

Hydrogen bonding has been described by chemists and crystallographers in a variety of ways, but a recent article defines it as "an attractive interaction between a hydrogen atom from a molecule or a molecular fragment $\mathrm{X}-\mathrm{H}$ in which $\mathrm{X}$ is more electronegative than $\mathrm{H}$, and an atom or a group of atoms in the same or a different molecule, in which there is evidence of bond formation". ${ }^{36}$ Intermolecular hydrogen bond interaction is the most important interaction type because it combines strength with directionality and in crystal engineering, it plays a key role in molecular association with biological systems. It acts as a key to predictingthe structural features of enzymes and proteins. ${ }^{37}$ Geometrically, the hydrogen bond X-H...A is usually described by three parameters, viz. $\boldsymbol{d}(\mathrm{H} \ldots \mathrm{A}), \boldsymbol{D}(\mathrm{X} \ldots \mathrm{A})$, and angle $\boldsymbol{\theta}(\mathrm{X}-\mathrm{H} \ldots \mathrm{A})$, to help analyze the strength and directionality of the hydrogen bond interactions. A classical hydrogen bond places the bridging proton squarely between a proton-donor and acceptor atom, as might occur, for example, between the two $\mathrm{O}$ atoms of the water dimer. While such geometry might, in fact, represent a preferred structure, there have been numerous observations of what has come to be termed bifurcated hydrogen bonds. The Halogen bonds are influenced by the surrounding media and the solvent indeed plays a role, opening the door for a multitude of applications, including, for instance, the design of macromolecular hosts capable of recognizing and binding anions. Since a great deal has been learned about the halogen bond in recent years and its parallels to the hydrogen bond, we investigated these interactions with intent to (i) find out the number and nature of hydrogen bonding present in the chlorine substituted quinoline derivatives and strength of these interactions, (ii) understand the role of hydrogen bond interactions in crystal structures and (iii) analyze different types of H-bonded interactions by employing graphical means.

\section{Intermolecular Hydrogen Bonding}

The bonds between independent molecules in a crystal structure are termed intermolecular hydrogen bonds. They play a vital role in lending stability to molecular packing in the unit cell. In order to compute and analyze intermolecular interactions in each molecule (M-1 to M-26), the Mercury software ${ }^{38}$ was used and the interactions thus observed are presented in Table-5. 
RASĀYAN J. Chem.

47-63| Special Issue | 2021

Table-5: The Geometry of Intermolecular Hydrogen Bonding Interactions

\begin{tabular}{|c|c|c|c|c|}
\hline S. No. & X-H....A & $\mathrm{d}(\mathrm{H} \ldots \mathrm{A}) \AA$ & $\mathrm{D}(\mathrm{X} \ldots . . \mathrm{A}) \AA$ & $\Theta(\mathrm{X}-\mathrm{H} \ldots \mathrm{A})^{\circ}$ \\
\hline $\begin{array}{c}\text { M-1 } \\
\text { Donor }=5 \\
\text { Acceptor }=3\end{array}$ & $\begin{array}{c}\text { N2A-H2A } 1 \ldots \text { N3B } \\
\text { N3A-H3A...Cl1A } \\
\text { N3A-H3B ...Cl1B } \\
\text { C10B-H10F ...Cl1A } \\
\text { C5B-H5B...Cl1A }\end{array}$ & $\begin{array}{l}2.230 \\
2.728 \\
2.860 \\
2.911 \\
2.930\end{array}$ & $\begin{array}{l}3.012 \\
3.337 \\
3.527 \\
3.601 \\
3.588\end{array}$ & $\begin{array}{l}152.20 \\
126.93 \\
132.73 \\
128.32 \\
127.53\end{array}$ \\
\hline $\begin{array}{c}\mathrm{M}-2 \\
\text { Donor }=4 \\
\text { Acceptor }=3\end{array}$ & $\begin{array}{c}\text { C9-H9B...N1 } \\
\text { C6-H6...O2 } \\
\text { C10-H10...O2 } \\
\text { C9-H9A...C9 }\end{array}$ & $\begin{array}{l}2.675 \\
2.623 \\
2.559 \\
2.891 \\
\end{array}$ & $\begin{array}{l}3.542 \\
3.429 \\
3.389 \\
3.827 \\
\end{array}$ & $\begin{array}{l}150.55 \\
145.46 \\
148.88 \\
165.23 \\
\end{array}$ \\
\hline $\begin{array}{c}\text { M-3 } \\
\text { Donor }=4 \\
\text { Acceptor }=4\end{array}$ & $\begin{array}{c}\mathrm{C} 4-\mathrm{H} 4 \ldots \mathrm{Cl} 1 \\
\mathrm{C} 5-\mathrm{H} 5 \ldots \mathrm{Cl1} \\
\mathrm{C} 10-\mathrm{H} 10 \mathrm{~B} \ldots \mathrm{Cl} 2 \\
\mathrm{C} 7-\mathrm{H} 7 \ldots \mathrm{C} 7 \\
\mathrm{C} 7-\mathrm{H} 7 \ldots \mathrm{C} 8\end{array}$ & $\begin{array}{l}2.759 \\
2.850 \\
2.780 \\
2.718 \\
2.855\end{array}$ & $\begin{array}{l}3.680 \\
3.763 \\
3.866 \\
3.786 \\
3.828 \\
\end{array}$ & $\begin{array}{l}143.38 \\
142.61 \\
173.11 \\
171.21 \\
150.27\end{array}$ \\
\hline $\begin{array}{c}\mathrm{M}-4 \\
\text { Donor }=3 \\
\text { Acceptor }=4\end{array}$ & 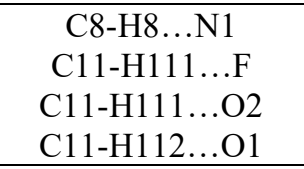 & $\begin{array}{l}2.622 \\
2.614 \\
2.652 \\
2.610 \\
\end{array}$ & $\begin{array}{l}3.526 \\
3.505 \\
3.334 \\
3.199 \\
\end{array}$ & $\begin{array}{l}160.63 \\
161.99 \\
131.16 \\
118.73 \\
\end{array}$ \\
\hline $\begin{array}{c}\text { M-5 } \\
\text { Donor }=1 \\
\text { Acceptor }=1\end{array}$ & C10-H10A...Cl1 & 2.867 & 3.745 & 151.16 \\
\hline $\begin{array}{c}\text { M-6 } \\
\text { Donor }=1 \\
\text { Acceptor }=1\end{array}$ & C7-H7 ...N1 & 2.613 & 3.530 & 169.00 \\
\hline $\begin{array}{c}\text { M-7 } \\
\text { Donor }=5 \\
\text { Acceptor }=4\end{array}$ & $\begin{array}{c}\mathrm{O} 1-\mathrm{H} 1 \mathrm{O} \ldots \mathrm{N} 1 \\
\mathrm{O} 2-\mathrm{H} 2 \mathrm{O} \ldots \mathrm{N} 2 \\
\mathrm{C} 11-\mathrm{H} 11 \mathrm{C} \ldots \mathrm{N} 1 \\
\mathrm{C} 11-\mathrm{H} 11 \mathrm{~A} \ldots \mathrm{O} 1 \\
\mathrm{C} 15-\mathrm{H} 15 \ldots \mathrm{O} 2\end{array}$ & $\begin{array}{l}2.152 \\
2.211 \\
2.678 \\
2.651 \\
2.691\end{array}$ & $\begin{array}{l}2.922 \\
2.978 \\
3.609 \\
3.421 \\
3.454\end{array}$ & $\begin{array}{l}161.08 \\
159.95 \\
163.47 \\
137.49 \\
140.09\end{array}$ \\
\hline $\begin{array}{c}\text { M-8 } \\
\text { Donor }=5 \\
\text { Acceptor }=4\end{array}$ & $\begin{array}{c}\text { N4-H4 ... } 11 \\
\mathrm{C} 8-\mathrm{H} 8 \ldots \mathrm{Cl} 1 \\
\mathrm{C} 9-\mathrm{H} 9 \ldots \mathrm{Cl} 2 \\
\mathrm{C} 10-\mathrm{H} 10 \ldots \mathrm{Cl} 1 \\
\mathrm{C} 11-\mathrm{H} 11 \ldots \mathrm{C} 10\end{array}$ & $\begin{array}{l}2.608 \\
2.827 \\
2.864 \\
2.780 \\
2.839 \\
\end{array}$ & $\begin{array}{l}3.521 \\
3.897 \\
3.878 \\
3.816 \\
3.867 \\
\end{array}$ & $\begin{array}{l}141.72 \\
167.84 \\
156.15 \\
160.60 \\
158.31 \\
\end{array}$ \\
\hline $\begin{array}{c}\text { M-9 } \\
\text { Donor }=2 \\
\text { Acceptor }=1\end{array}$ & $\begin{array}{l}\mathrm{C} 4-\mathrm{H} 4 \ldots \mathrm{O} 1 \\
\mathrm{C} 5-\mathrm{H} 5 \ldots \mathrm{O} 1\end{array}$ & $\begin{array}{l}2.604 \\
2.634\end{array}$ & $\begin{array}{l}3.436 \\
3.453\end{array}$ & $\begin{array}{l}149.09 \\
147.19\end{array}$ \\
\hline $\begin{array}{c}\text { M-10 } \\
\text { Donor }=4 \\
\text { Acceptor }=4\end{array}$ & $\begin{array}{c}\mathrm{C} 8-\mathrm{H} 8 \ldots \mathrm{N} 1 \\
\mathrm{C} 10-\mathrm{H} 10 \ldots \mathrm{O} 2 \\
\mathrm{C} 7-\mathrm{H} 7 \ldots \mathrm{O} 1 \\
\mathrm{C} 4-\mathrm{H} 4 \ldots \mathrm{C} 11 \\
\end{array}$ & $\begin{array}{l}2.569 \\
2.516 \\
2.708 \\
2.830 \\
\end{array}$ & $\begin{array}{l}3.498 \\
3.403 \\
3.619 \\
3.754 \\
\end{array}$ & $\begin{array}{l}176.13 \\
159.54 \\
166.70 \\
172.23 \\
\end{array}$ \\
\hline $\begin{array}{c}\text { M-11 } \\
\text { Donor }=2 \\
\text { Acceptor }=1\end{array}$ & $\begin{array}{l}\text { C4-H4 ...O1 } \\
\text { C6-H6 ...O1 }\end{array}$ & $\begin{array}{l}2.643 \\
2.720\end{array}$ & $\begin{array}{l}3.493 \\
3.560\end{array}$ & $\begin{array}{l}152.35 \\
150.64\end{array}$ \\
\hline $\begin{array}{c}\text { M-12 } \\
\text { Donor }=3 \\
\text { Acceptor }=3\end{array}$ & $\begin{array}{c}\mathrm{C} 4-\mathrm{H} 4 \ldots \mathrm{O} 1 \\
\mathrm{C} 5-\mathrm{H} 5 \ldots \mathrm{O} 1 \\
\mathrm{C} 11-\mathrm{H} 11 \mathrm{~A} \ldots \mathrm{O} 2 \\
\mathrm{C} 11-\mathrm{H} 11 \mathrm{~A} \ldots \mathrm{N} 1 \\
\end{array}$ & $\begin{array}{l}2.667 \\
2.706 \\
2.635 \\
2.665 \\
\end{array}$ & $\begin{array}{l}3.504 \\
3.523 \\
3.361 \\
3.598 \\
\end{array}$ & $\begin{array}{l}150.09 \\
147.08 \\
132.73 \\
163.66 \\
\end{array}$ \\
\hline $\begin{array}{c}\text { M-13 } \\
\text { Donor }=1 \\
\text { Acceptor }=1\end{array}$ & C11-H11A...O1 & 2.536 & 3.467 & 163.73 \\
\hline $\begin{array}{c}\text { M-14 } \\
\text { Donor }=1 \\
\text { Acceptor }=1\end{array}$ & C7-H7 ...O1 & 2.694 & 3.489 & 142.94 \\
\hline
\end{tabular}


RASĀYAN J. Chem.

47-63| Special Issue | 2021

\begin{tabular}{|c|c|c|c|c|}
\hline $\begin{array}{c}\text { M-15 } \\
\text { Donor }=4 \\
\text { Acceptor }=3\end{array}$ & $\begin{array}{l}\mathrm{O} 2-\mathrm{H} 2 \ldots \mathrm{N} 1 \\
\mathrm{C} 8-\mathrm{H} 8 \ldots \mathrm{O} 1 \\
\mathrm{C} 6-\mathrm{H} 6 \ldots \mathrm{O} 1 \\
\mathrm{C} 4-\mathrm{H} 4 \ldots \mathrm{C} 6\end{array}$ & $\begin{array}{l}1.950 \\
2.370 \\
2.635 \\
2.891\end{array}$ & $\begin{array}{l}2.768 \\
3.290 \\
3.226 \\
3.660\end{array}$ & $\begin{array}{l}164.00 \\
163.00 \\
120.79 \\
138.77\end{array}$ \\
\hline $\begin{array}{c}\text { M-16 } \\
\text { Donor }=2 \\
\text { Acceptor }=2\end{array}$ & 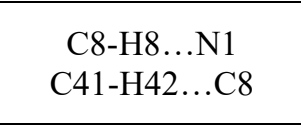 & $\begin{array}{l}2.613 \\
2.756\end{array}$ & $\begin{array}{l}3.517 \\
3.606\end{array}$ & $\begin{array}{l}160.00 \\
145.34\end{array}$ \\
\hline $\begin{array}{c}\text { M-17 } \\
\text { Donor =1 } \\
\text { Acceptor = } 1\end{array}$ & C11A-H11F...Cl2B & 2.774 & 3.701 & 162.65 \\
\hline $\begin{array}{c}\text { M-18 } \\
\text { Donor }=4 \\
\text { Acceptor }=4\end{array}$ & $\begin{array}{c}\text { C5A-H5A...Cl2 } \\
\text { C11B-H11AB ...N1 } \\
\text { C5B-H5B...C14 } \\
\text { C11B-H11AB...N2 }\end{array}$ & $\begin{array}{l}2.732 \\
2.410 \\
2.763 \\
2.251 \\
\end{array}$ & $\begin{array}{l}3.094 \\
2.825 \\
3.135 \\
2.744 \\
\end{array}$ & $\begin{array}{l}104.01 \\
106.00 \\
105.20 \\
111.00\end{array}$ \\
\hline $\begin{array}{c}\text { M-19 } \\
\text { Donor }=2 \\
\text { Acceptor }=2\end{array}$ & $\begin{array}{c}\mathrm{C} 3-\mathrm{H} 3 \ldots \mathrm{F} 2 \\
\mathrm{C} 4-\mathrm{H} 4 \ldots \mathrm{C} 11\end{array}$ & $\begin{array}{l}2.663 \\
2.759\end{array}$ & $\begin{array}{l}3.428 \\
3.661\end{array}$ & $\begin{array}{l}138.00 \\
158.88\end{array}$ \\
\hline $\begin{array}{c}\text { M-20 } \\
\text { Donor }=2 \\
\text { Acceptor }=2\end{array}$ & $\begin{array}{l}\mathrm{C} 8-\mathrm{H} 8 \ldots \mathrm{N} 1 \\
\mathrm{C} 3-\mathrm{H} 3 \ldots \mathrm{F} 2\end{array}$ & $\begin{array}{l}2.695 \\
2.623\end{array}$ & $\begin{array}{l}3.607 \\
3.243\end{array}$ & $\begin{array}{l}166.85 \\
124.63\end{array}$ \\
\hline $\begin{array}{c}\mathrm{M}-21 \\
\text { Donor }=2 \\
\text { Acceptor }=2\end{array}$ & $\begin{array}{l}\mathrm{N} 2-\mathrm{H} 2 \ldots \mathrm{N} 1 \\
\mathrm{C} 8-\mathrm{H} 8 \ldots \mathrm{C} 8\end{array}$ & $\begin{array}{l}2.301 \\
2.837\end{array}$ & $\begin{array}{l}3.142 \\
3.715\end{array}$ & $\begin{array}{l}171.16 \\
157.99\end{array}$ \\
\hline $\begin{array}{c}\text { M-22 } \\
\text { Donor }=1 \\
\text { Acceptor }=1\end{array}$ & $\mathrm{~N} 2-\mathrm{H} 2 \ldots \mathrm{N} 1$ & 2.253 & 3.051 & 154.48 \\
\hline $\begin{array}{c}\text { M-23 } \\
\text { Donor }=1 \\
\text { Acceptor }=1\end{array}$ & $\mathrm{~N} 2-\mathrm{H} 2 \ldots \mathrm{N} 2$ & 2.530 & 3.353 & 165.24 \\
\hline $\begin{array}{c}\text { M-24 } \\
\text { Donor }=3 \\
\text { Acceptor }=3\end{array}$ & $\begin{array}{l}\text { C3A-H3AA...Cl4 } \\
\text { C7B-H7BA...N1 } \\
\text { C8B-H8BA...Cl1 }\end{array}$ & $\begin{array}{l}2.883 \\
2.600 \\
2.950\end{array}$ & $\begin{array}{l}3.719 \\
3.511 \\
3.729\end{array}$ & $\begin{array}{l}148.30 \\
161.96 \\
140.50\end{array}$ \\
\hline $\begin{array}{c}\mathrm{M}-25 \\
\text { Donor }=1 \\
\text { Acceptor }=1\end{array}$ & O1-H1O_..O1 & 1.895 & 2.712 & 173.92 \\
\hline $\begin{array}{c}\text { M-26 } \\
\text { Donor }=2 \\
\text { Acceptor }=2\end{array}$ & $\begin{array}{c}\mathrm{O} 1-\mathrm{H} 1 \mathrm{O} \ldots \mathrm{O} 1 \\
\mathrm{C} 4-\mathrm{H} 4 \ldots \mathrm{Cl} 1\end{array}$ & $\begin{array}{l}1.929 \\
2.866\end{array}$ & $\begin{array}{l}2.716 \\
3.781\end{array}$ & $\begin{array}{l}176.89 \\
167.74\end{array}$ \\
\hline
\end{tabular}

A variety of intermolecular H-bonded interactions of the type C-H..., C-H...Cl, C-H...N, C-H...C, N$\mathrm{H} . . \mathrm{N}, \mathrm{C}-\mathrm{H} \ldots \mathrm{F}, \mathrm{O}-\mathrm{H} \ldots \mathrm{N}, \mathrm{O}-\mathrm{H} \ldots \mathrm{O}, \mathrm{N}-\mathrm{H} \ldots \mathrm{Cl}, \mathrm{C}-\mathrm{H} \ldots \mathrm{Br}$ and $\mathrm{N}-\mathrm{H} . . \mathrm{F}$ have been observed. The frequency of occurrence of the stated class of interactions and their distance-angle scatter plots are presented in Fig.2 and 3, respectively. A critical analysis of the plots indicates that the C-H...O interactions are quite dominant (frequency of occurrence $\sim 27.5 \%$ ) and that it significantly contributes to the molecular packing. Based on the range of values as obtained in the case of the geometrical parameters, viz.d $[$ (H...A) $=2.37-2.72 \AA], \boldsymbol{D}[(\mathrm{X} \ldots \mathrm{A})=3.19-3.62 \AA]$ and $\boldsymbol{\theta}\left[(\mathrm{X}-\mathrm{H} \ldots \mathrm{A})=118-167^{\circ}\right]$, it is suggested that the C$\mathrm{H}$...O type intermolecular interactions lead to the category of weak $\mathrm{H}$-bonding. ${ }^{39}$ Further, the bifurcated $\mathrm{C}-\mathrm{H} \ldots \mathrm{O}$ interactions (with $\mathrm{O}$ being a bifurcated acceptor) are equally significant as they exist in quite a good number of structures (M-2, M-9, M-11, M-12 and M-15).

An illustration of the bifurcated $\mathrm{C}-\mathrm{H} . . . \mathrm{O}$ bond network (for M-11 and M-12) is shown in Fig.-4. The frequency of occurrence of halogenated hydrogen bond-like $\mathrm{C}-\mathrm{H} . . \mathrm{Cl}$ is equally significant (incidence of occurrence $\sim 23.2 \%$ ) and comparable with the corresponding population of $\mathrm{C}-\mathrm{H}$...O interactions. However, the range of values like $\boldsymbol{d}[(\mathrm{H} \ldots \mathrm{A})=2.73-2.95 \AA], \boldsymbol{D}[(\mathrm{X} \ldots \mathrm{A})=3.095-3.897 \AA]$ and $\boldsymbol{\theta}[(\mathrm{X}-\mathrm{H} \ldots \mathrm{A})$ $=104^{\circ}-173^{\circ}$, for halogen interactions further vindicate the findings as suggested above that such 
RASĀYAN J. Chem.

47-63| Special Issue | 2021

interactions are simply the weak hydrogen bonds ${ }^{39}$ but they do play a very significant role in molecular packing by forming molecular chains along with different unit cell directions (Fig.-5).

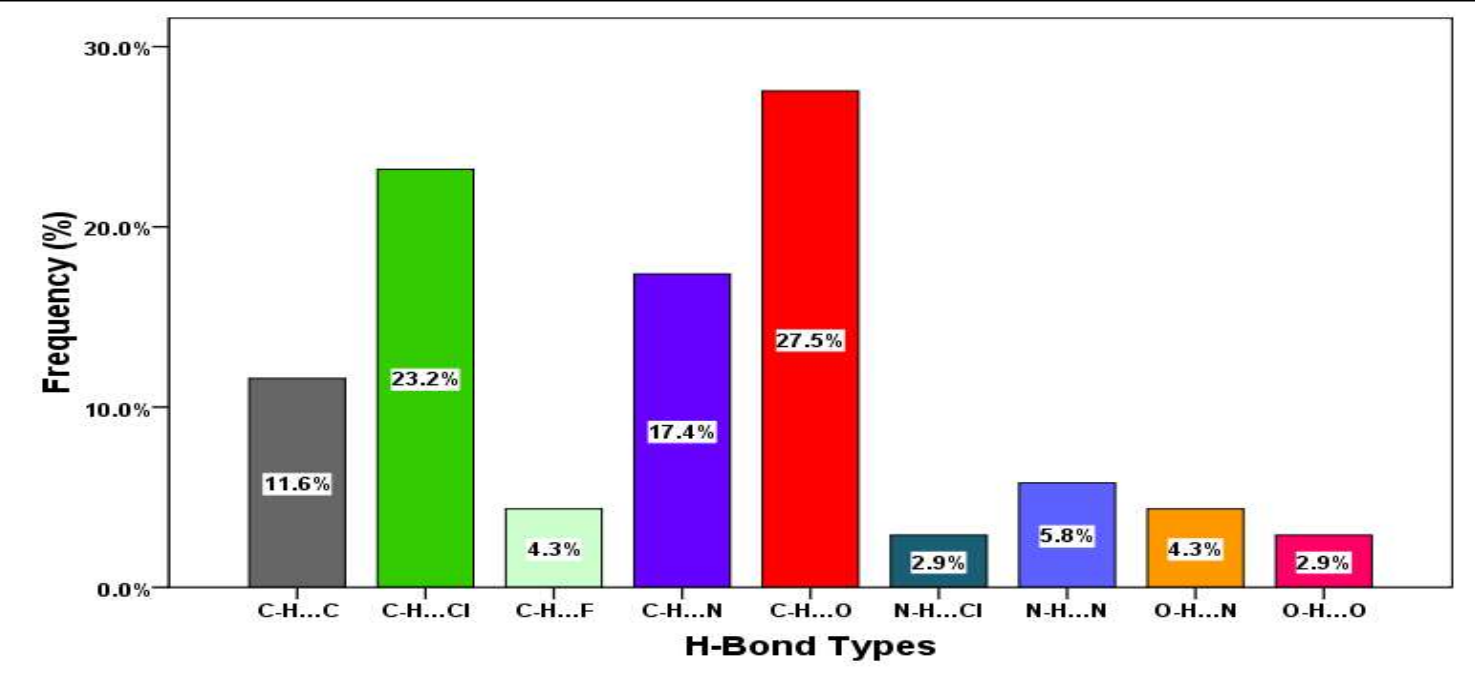

Fig.-2: Frequency of Occurrence of Various Types of Intermolecular Interactions

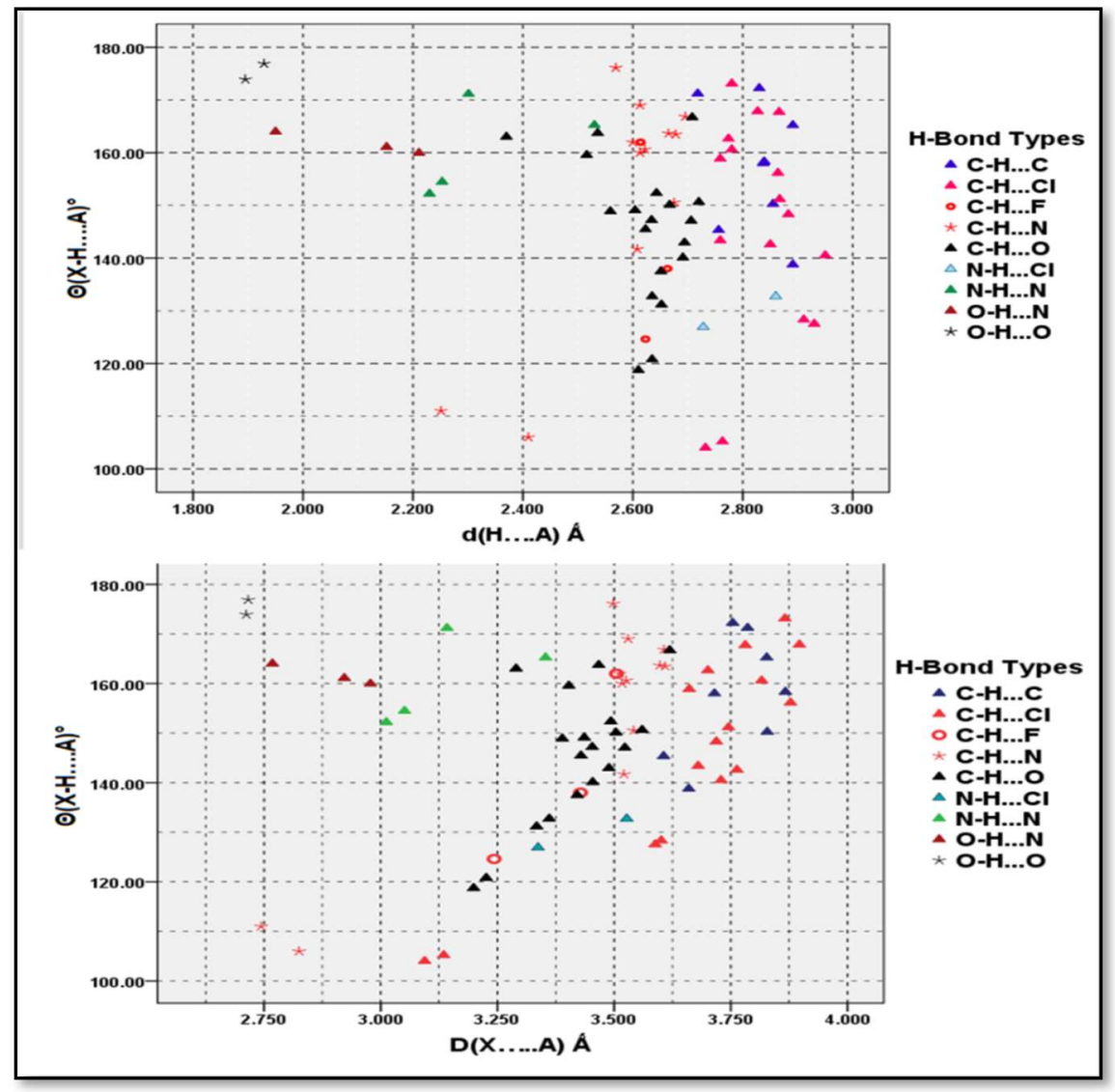

Fig.-3: Scattered Plots for d vs. X-H...A and D vs. X-H...A, in Intermolecular Interaction

The molecular packing for M-7 and M-15 is accompanied by a strong O-H...N network, while it is mainly stabilized by strong O-H...O bonds in the case of M-25 and M-26, respectively. A view of crystal packing involving $\mathrm{C}-\mathrm{H} \ldots \mathrm{O}, \mathrm{C}-\mathrm{H} \ldots \mathrm{N}$ and $\mathrm{C}-\mathrm{H} \ldots \mathrm{Cl}$ bonds forming three-dimensional networks in $\mathrm{M}-10$ 
RASĀYAN J. Chem.

47-63| Special Issue | 2021

are presented in Fig.-6. The number of other interactions $[\mathrm{C}-\mathrm{H} \ldots \mathrm{N}$ and $\mathrm{N}-\mathrm{H} \ldots \mathrm{N}, \mathrm{N}-\mathrm{H} \ldots \mathrm{Cl}, \mathrm{O}-\mathrm{H} \ldots \mathrm{N}]$ is too small to merit any specific consideration.

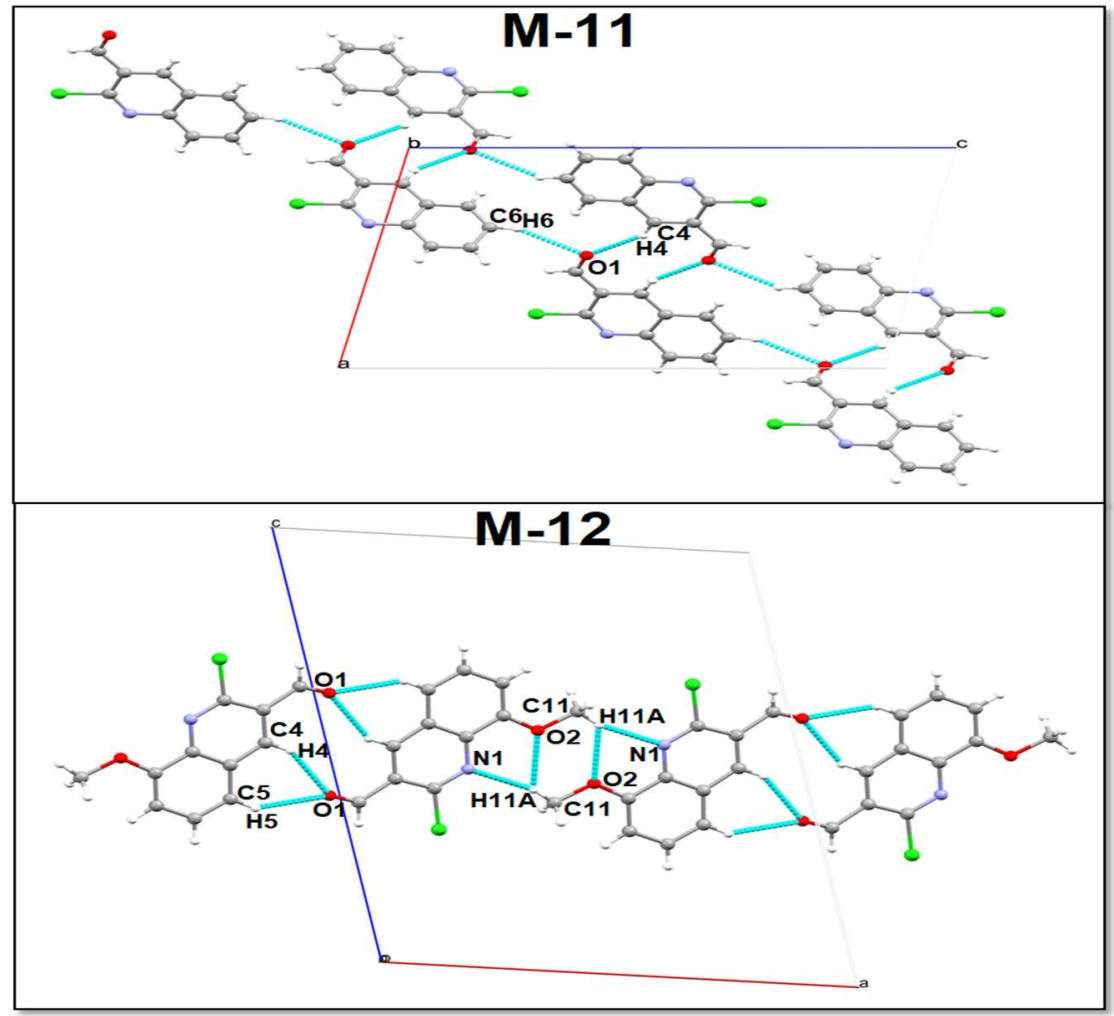

Fig.-4: View of C-H...O bifurcated H-bonding network in $\mathrm{M}-11^{17}$ and $\mathrm{M}-12^{18}$

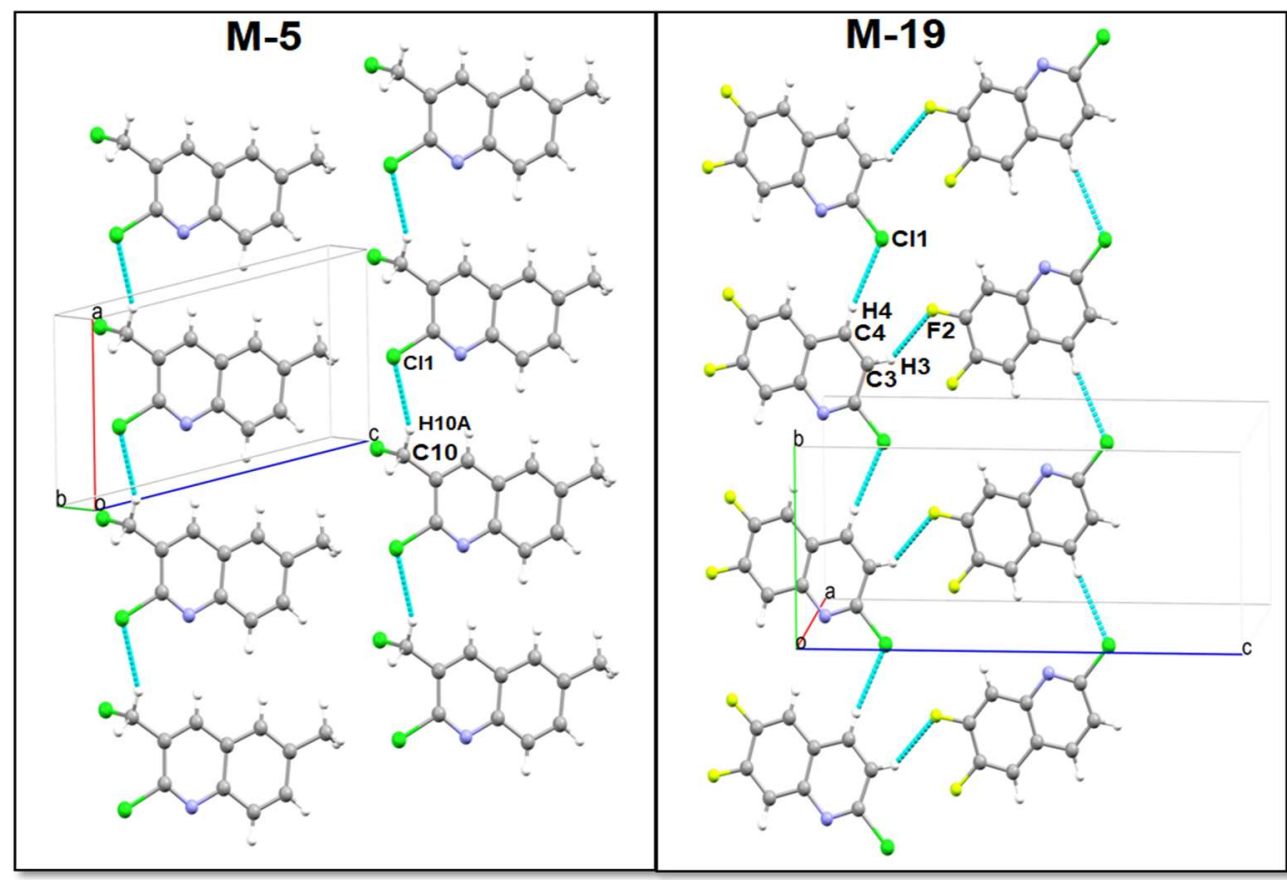

Fig.-5: Formation of C-H...Cl Chains in $\mathrm{M}-5^{13}$ and $\mathrm{M}-19^{25}$ 
RASĀYAN J. Chem.

47-63| Special Issue | 2021

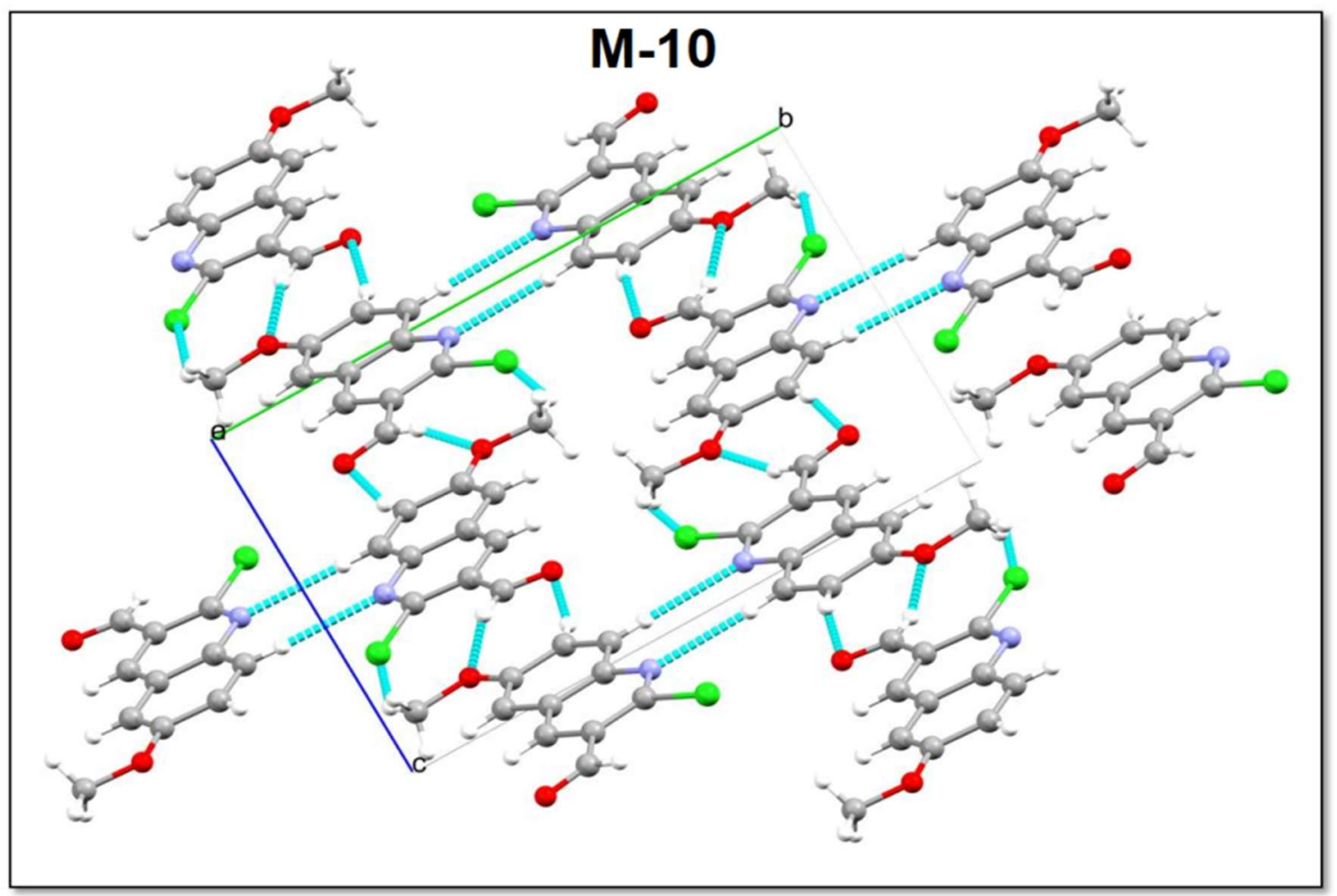

Fig.-6: Molecular Packing involving C-H...O, C-H...N and C-H...Cl H-bonds in M-10 ${ }^{16}$

\section{Intramolecular Hydrogen Bonding}

The intramolecular hydrogen bond is said to be happening within the isolated molecule and such bonds are reportedly useful in the stability of molecular conformations. ${ }^{40} \mathrm{~A}$ great deal of intra-molecular $\mathrm{H}$ bonded interactions, viz. C-H...Cl, C-H...O, C-H...C, N-H...F, C-H...N, C-H...F, N-H...Cl and N$\mathrm{H} \ldots \mathrm{N}$, have been observed in such molecules and their geometrical parameters and geometrical range for $\boldsymbol{d}[(\mathrm{H} . . \mathrm{A})=(1.392-2.796) \AA], \boldsymbol{D}[(\mathrm{X} \ldots \mathrm{A})=(2.684-3.114) \AA]$ and $\boldsymbol{\theta}\left[(\mathrm{X}-\mathrm{H} \ldots \mathrm{A})=96^{\circ}-117.13^{\circ}\right]$ are presented in Table-6.

Table-6: The Geometry of Intramolecular Hydrogen Bonding Interactions

\begin{tabular}{|c|c|c|c|c|}
\hline S.No. & X-H....A & $\mathrm{d}(\mathrm{H} \ldots \mathrm{A}) \mathrm{A})$ & $\mathrm{D}(\mathrm{X} \ldots \ldots \mathrm{A}) \hat{A}$ & $\Theta(X-H \ldots . . A)^{\circ}$ \\
\hline $\begin{array}{c}\mathrm{M}-2 \\
\text { Donor }=3 \\
\text { Acceptor }=2\end{array}$ & $\begin{array}{c}\mathrm{C} 11-\mathrm{H} 11 \ldots \mathrm{Cl} 1 \\
\mathrm{C} 3-\mathrm{H} 3 \ldots \mathrm{C} 1 \\
\mathrm{C} 10-\mathrm{H} 10 \ldots \mathrm{O} 2\end{array}$ & $\begin{array}{l}2.746 \\
2.500 \\
2.572\end{array}$ & $\begin{array}{l}3.088 \\
2.793 \\
2.838\end{array}$ & $\begin{array}{c}102.18 \\
98.51 \\
96.87\end{array}$ \\
\hline $\begin{array}{c}\text { M-3 } \\
\text { Donor }=1 \\
\text { Acceptor }=1\end{array}$ & C10-H10A...Cl1 & 2.686 & 3.054 & 99.07 \\
\hline $\begin{array}{c}\mathrm{M}-4 \\
\text { Donor }=2 \\
\text { Acceptor }=2\end{array}$ & $\begin{array}{c}\mathrm{C} 1-\mathrm{H} 1 \ldots \mathrm{Cl} \\
\mathrm{C} 7-\mathrm{H} 7 \ldots \mathrm{C} 11\end{array}$ & $\begin{array}{l}2.738 \\
2.521\end{array}$ & $\begin{array}{l}3.131 \\
2.825\end{array}$ & $\begin{array}{c}105.63 \\
98.46\end{array}$ \\
\hline $\begin{array}{c}\text { M-5 } \\
\text { Donor }=1 \\
\text { Acceptor }=1\end{array}$ & C10-H10B...Cl1 & 2.725 & 3.063 & 101.35 \\
\hline $\begin{array}{c}\text { M-8 } \\
\text { Donor }=2 \\
\text { Acceptor }=2\end{array}$ & $\begin{array}{c}\mathrm{C} 8-\mathrm{H} 8 \ldots \mathrm{C} 10 \\
\mathrm{C} 10-\mathrm{H} 10 \ldots \mathrm{C} 8\end{array}$ & $\begin{array}{l}2.419 \\
1.392\end{array}$ & $\begin{array}{l}2.960 \\
2.960\end{array}$ & $\begin{array}{l}109.42 \\
111.20\end{array}$ \\
\hline $\begin{array}{c}\text { M-9 } \\
\text { Donor }=2\end{array}$ & $\begin{array}{c}\mathrm{C} 10-\mathrm{H} 10 \ldots \mathrm{Cl} 1 \\
\mathrm{C} 3-\mathrm{H} 3 \ldots \mathrm{O} 1\end{array}$ & $\begin{array}{l}2.689 \\
2.571\end{array}$ & $\begin{array}{l}3.088 \\
2.842\end{array}$ & $\begin{array}{c}106.77 \\
97.25\end{array}$ \\
\hline
\end{tabular}


RASĀYAN J. Chem.

47-63| Special Issue | 2021

\begin{tabular}{|c|c|c|c|c|}
\hline Acceptor $=2$ & & & & \\
\hline $\begin{array}{c}\text { M-10 } \\
\text { Donor }=2 \\
\text { Acceptor }=2\end{array}$ & $\begin{array}{c}\mathrm{C} 10-\mathrm{H} 10 \ldots \mathrm{Cl} 1 \\
\mathrm{C} 3-\mathrm{H} 3 \ldots \mathrm{O} 1\end{array}$ & $\begin{array}{l}1.739 \\
2.539\end{array}$ & $\begin{array}{l}3.125 \\
2.825\end{array}$ & $\begin{array}{c}105.99 \\
98.18\end{array}$ \\
\hline $\begin{array}{c}\text { M-11 } \\
\text { Donor }=2 \\
\text { Acceptor }=2\end{array}$ & $\begin{array}{c}\mathrm{C} 10-\mathrm{H} 10 \ldots \mathrm{Cl} 1 \\
\mathrm{C} 3-\mathrm{H} 3 \ldots \mathrm{O} 1\end{array}$ & $\begin{array}{l}2.703 \\
2.571\end{array}$ & $\begin{array}{l}3.100 \\
2.841\end{array}$ & $\begin{array}{c}106.63 \\
97.13\end{array}$ \\
\hline $\begin{array}{c}\mathrm{M}-12 \\
\text { Donor }=2 \\
\text { Acceptor }=2\end{array}$ & $\begin{array}{c}\mathrm{C} 10-\mathrm{H} 10 \ldots \mathrm{Cl1} \\
\mathrm{C} 7-\mathrm{H} 7 \ldots \mathrm{C} 11\end{array}$ & $\begin{array}{l}2.730 \\
2.545\end{array}$ & $\begin{array}{l}3.117 \\
2.836\end{array}$ & $\begin{array}{c}106.04 \\
98.54\end{array}$ \\
\hline $\begin{array}{c}\text { M-13 } \\
\text { Donor }=2 \\
\text { Acceptor }=2\end{array}$ & $\begin{array}{c}\mathrm{C} 10-\mathrm{H} 10 \ldots \mathrm{Cl1} \\
\mathrm{C} 3-\mathrm{H} 3 \ldots \mathrm{O} 1\end{array}$ & $\begin{array}{l}2.688 \\
2.577\end{array}$ & $\begin{array}{l}3.095 \\
2.850\end{array}$ & $\begin{array}{c}107.36 \\
97.41\end{array}$ \\
\hline $\begin{array}{c}\text { M-14 } \\
\text { Donor }=1 \\
\text { Acceptor }=1\end{array}$ & C10-H10...Cl1 & 2.703 & 3.084 & 105.46 \\
\hline $\begin{array}{c}\text { M-15 } \\
\text { Donor }=1 \\
\text { Acceptor }=1\end{array}$ & $\mathrm{C} 3-\mathrm{H} 3 \ldots \mathrm{O} 2$ & 2.337 & 2.684 & 100.90 \\
\hline $\begin{array}{c}\text { M-16 } \\
\text { Donor }=1 \\
\text { Acceptor }=1\end{array}$ & $\mathrm{C} 5-\mathrm{H} 5 \ldots \mathrm{C} 41$ & 2.679 & 3.003 & 100.63 \\
\hline $\begin{array}{c}\text { M-17 } \\
\text { Donor }=1 \\
\text { Acceptor }=1\end{array}$ & C5A-H5AA...Cl2A & 2.704 & 3.092 & 104.79 \\
\hline $\begin{array}{c}\text { M-18 } \\
\text { Donor }=2 \\
\text { Acceptor }=2\end{array}$ & $\begin{array}{c}\mathrm{C} 5-\mathrm{H} 5 \ldots \mathrm{Cl} 2 \\
\mathrm{C} 11-\mathrm{H} 11 \mathrm{~B} \ldots \mathrm{N} 1\end{array}$ & $\begin{array}{l}2.727 \\
2.405\end{array}$ & $\begin{array}{l}3.094 \\
2.825\end{array}$ & $\begin{array}{l}104.49 \\
106.04\end{array}$ \\
\hline $\begin{array}{c}\text { M-20 } \\
\text { Donor }=1 \\
\text { Acceptor }=1\end{array}$ & $\mathrm{C} 4-\mathrm{H} 4 \ldots \mathrm{F} 1$ & 2.552 & 2.817 & 96.81 \\
\hline $\begin{array}{c}\text { M-21 } \\
\text { Donor }=1 \\
\text { Acceptor }=1\end{array}$ & $\mathrm{~N} 2-\mathrm{H} 2 \ldots \mathrm{F} 1$ & 2.357 & 2.699 & 104.44 \\
\hline $\begin{array}{c}\text { M-22 } \\
\text { Donor }=1 \\
\text { Acceptor }=1\end{array}$ & $\mathrm{~N} 2-\mathrm{H} 2 \ldots \mathrm{Cl} 2$ & 2.555 & 2.961 & 109.93 \\
\hline $\begin{array}{c}\text { M-23 } \\
\text { Donor }=1 \\
\text { Acceptor }=1\end{array}$ & $\mathrm{~N} 2-\mathrm{H} 2 \ldots \mathrm{N} 1$ & 2.343 & 2.700 & 105.92 \\
\hline $\begin{array}{c}\text { M-24 } \\
\text { Donor }=1 \\
\text { Acceptor }=1\end{array}$ & C5-H5A...Cl2 & 2.740 & 3.114 & 104.32 \\
\hline
\end{tabular}

Among the various intra-molecular interactions, the concentration of $\mathrm{C}-\mathrm{H} . . \mathrm{Cl}$ type interactions are quite large $(41.38 \%)$ and that of $\mathrm{C}-\mathrm{H} . . \mathrm{O}$ and $\mathrm{C}-\mathrm{H} . . . \mathrm{C}$ bonds is $20.69 \%$ each. The incidence of occurrence for $\mathrm{N}-\mathrm{H} \ldots \mathrm{N}, \mathrm{N}-\mathrm{H} \ldots \mathrm{Cl}, \mathrm{O}-\mathrm{H} \ldots \mathrm{N}, \mathrm{N}-\mathrm{H} \ldots \mathrm{F}, \mathrm{C}-\mathrm{H} \ldots \mathrm{F}$ and $\mathrm{C}-\mathrm{H} \ldots \mathrm{N}$ type of interactions is quite low, though not 
RASĀYAN J. Chem.

47-63 | Special Issue | 2021

insignificant (Fig.-7). From the $\boldsymbol{d} \boldsymbol{\theta} \boldsymbol{\theta}$ scatter plots, viz. $\boldsymbol{d}(\mathrm{H} \ldots \mathrm{A})$ vs $\boldsymbol{\theta}(\mathrm{X}-\mathrm{H} \ldots \mathrm{A})$ and $\boldsymbol{D}(\mathrm{X} \ldots \mathrm{A})$ vs $\boldsymbol{\theta}(\mathrm{X}-$ H...A), for intra-molecular H-bonds(Fig.-8), maximum spot density has been observed in case of C$\mathrm{H} . . \mathrm{Cl}$ type interactions, followed by $\mathrm{C}-\mathrm{H} . . \mathrm{C}$ intramolecular interactions. A substantial number of halogen-based intramolecular $\mathrm{C}-\mathrm{H} \ldots \mathrm{Cl}$ and $\mathrm{C}-\mathrm{H} \ldots \mathrm{C}$ bonds are found to exist in the range $[\boldsymbol{d}(\mathrm{H} \ldots \mathrm{A})=$ $2.700 \AA \hat{-}-2.750 \AA$ and $\boldsymbol{D}(\mathrm{X} \ldots \mathrm{A})=3.000 \AA \hat{-}-3.200 \AA \hat{\AA}$ for $\boldsymbol{\theta}(\mathrm{X}-\mathrm{H} \ldots \mathrm{A})$ range $\left.\sim 97^{\circ}-108^{\circ}\right]$ and $[\boldsymbol{d}(\mathrm{H} \ldots \mathrm{A})=$

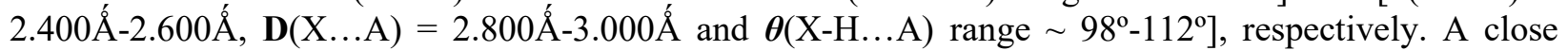
examination of their geometrical parameters conveniently places these interactions in the category of weak hydrogen bonds. ${ }^{41}$

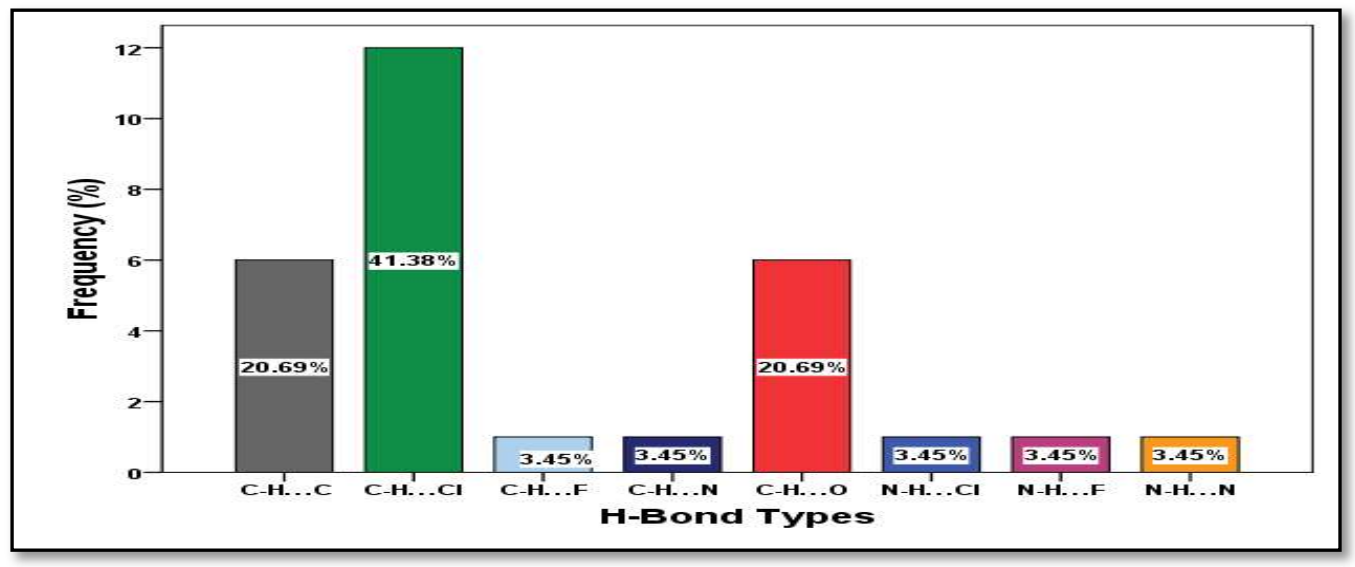

Fig.-7: Frequency of Occurrence of Various Types of Intramolecular Interaction

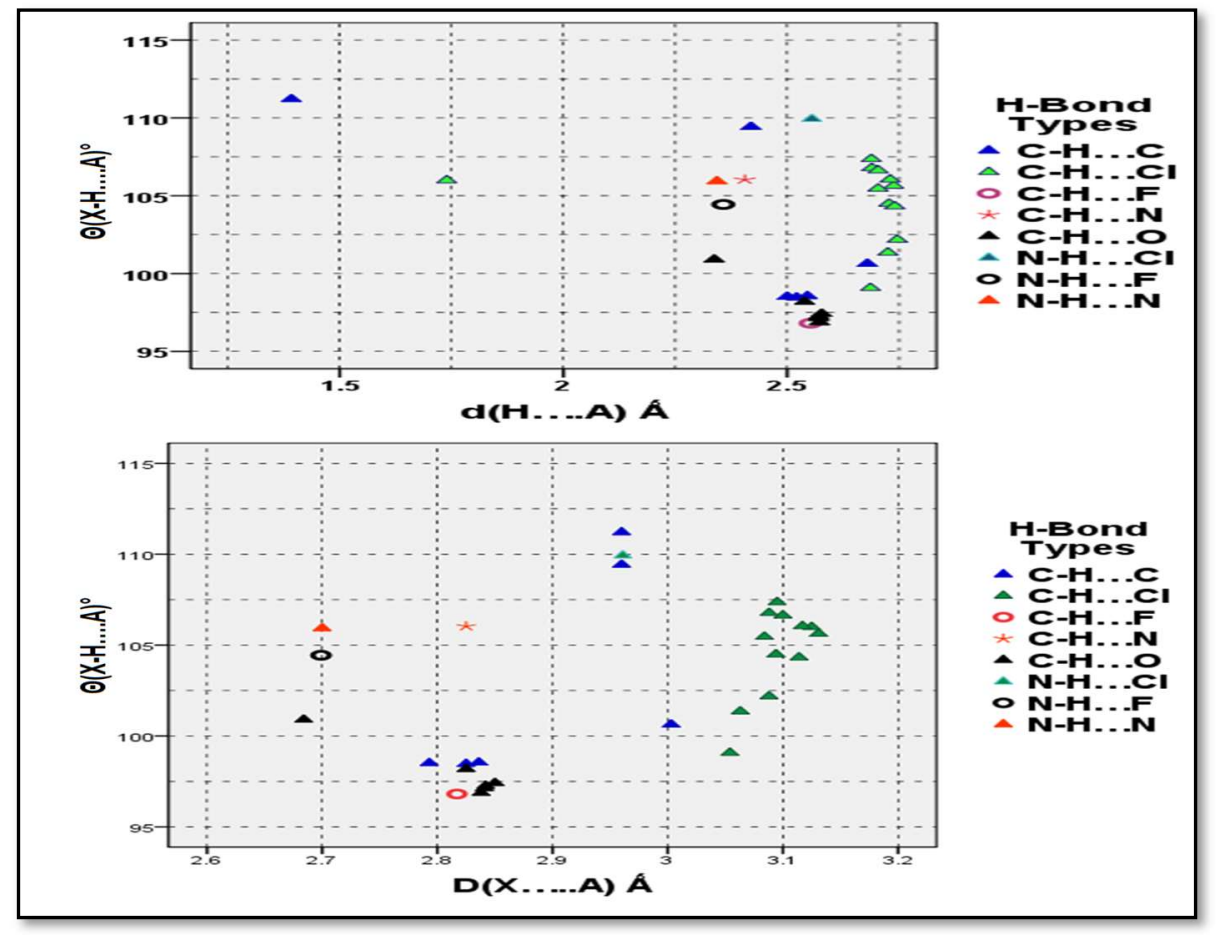

Fig.-8: Scatter Plots for d vs. X-H...A and D vs. X-H...A, in Intramolecular Interaction

CONCLUSION

Preliminary crystallographic analysis shows that most of the chlorine quinolines prefer to crystallize in a monoclinic crystal system with $\mathrm{P} 2_{1} / \mathrm{n}$ space group. The variation observed in bond distances and bond angles around $\mathrm{C} 2$ is most likely due to the involvement of the chlorine atom at $\mathrm{C} 2$ position. The predictions for chlorine quinoline derivative possessing biological activities and their comparative analysis reveal that most of these derivatives are quite active for anti-inflammatory, membrane 
RASĀYAN J. Chem.

47-63| Special Issue | 2021

permeability inhibitor, antiviral, complement factor D inhibitor and phobic disorders treatment. Most of the structures in the present investigation are found to be quite rich in forming a sizeable number of interand intra-molecular $\mathrm{C}-\mathrm{H} . . . \mathrm{O}$ and $\mathrm{C}-\mathrm{H} . . . \mathrm{Cl}$ hydrogen bonds. A careful analysis of the scatter plots depicts that such interactions are weak hydrogen bonds contributing effectively to defining the crystal packing effects. It is pertinent to mention that the participation of $\mathrm{Cl}$ atom (at $\mathrm{C} 2$ ) leads to the formation of intra and inter-molecular hydrogen bonds in most of the structures and hence plays a significant role in the characterization of molecular packing of chlorine quinoline structures. Another important feature observed from the graphical presentations is the existence of bifurcated C-H...O hydrogen bonding in several structures of the halogenated quinoline structures. Since the work of this kind is going on to take into its fold some other related halogen quinoline derivatives, the findings, as such, thus be considered empirical till comprehensive research is compiled and reported.

\section{ACKNOWLEDGEMENT}

Rajni Kant is thankful to the University of Jammu for funding under RUSA 2.0 received from the Government of India.

\section{REFERENCES}

1. S.Srisung, T.Suksrichavalit, S.Prachayasittikul, S.Ruchirawat and V.Prachayasittikul, International Journal of Pharmacology, 9, 170(2013), https://doi.org/10.3923/ijp.2013.170.175

2. J.Kos, I.Zadrazilova, E.Nevin, M.Soral, T.Gonec, P.Kollar, M.Oravec, A.Coffey, J.O'Mahony, T.Liptaj, K.Kralova and J.Jampilek, Bioorganic and Medicinal Chemistry, 23, 4188(2015), https://doi.org/10.1016/j.bmc.2015.06.047

3. J.M.Tanzer, A.M.Slee, B.Kamay and E.Scheer, American Society for Microbiology, 13, 1044(1978), https://doi.org/10.1128/aac.13.6.1044

4. H.Jiang, J.E.Taggarta, X.Zhanga, D.M.Benbrook, S.E.Lind and W.Ding, Cancer Letters,312, 11(2011), https://doi.org/10.1016/j.canlet.2011.06.032

5. P.Hongmanee, K.Rukseree, B.Buabut, B.Somsri and P.Palittapongarnpim, Antimicrobial Agents and Chemotherapy,51, 1105(2007), https://doi.org/10.1128/AAC.01310-06

6. N.P.Sahu, C.Pal, N.B.Mandal, S.Banerjee, M.Raha, A.P.Kundu, A.Basu, M.Ghosh, K.Roy and S.Bandyopadhyay, Bioorgganic \& Medical Chemistry, 10, 1687(2002), https://doi.org/10.1016/s0968-0896(02)00046-9

7. E.S.Badawey and T.Kappe, European Journal of Medical Chemistry, 32, 815(1997), https://doi.org/10.1016/S0223-5234(99)80067-1

8. W.R.Baker, S.Cai, M.Dimitroff, L.Fang, K.K.Huh, D.R.Ryckman, X.Shang, R.M.Shawar and J.H. Therrien, Journal of Medical Chemistry, 47, 4693(2004), https://doi.org/10.1021/jm0497895

9. G.S.Kumar, M.Zeller, R.G.Gonnade and K.J.R.Prasad, Tetrahedron Letters, 55, 4240(2014), https://doi.org/10.1016/j.tetlet.2014.05.132

10. S.Mukhopadhyay, R.K.Gupta, A.Biswas, A.Kumar,M.Dubey, M.S.Hundal, D.S.Pandey, Dalton Transactions ,44,7118(2015), https://doi.org/10.1039/C4DT03778B

11. V.R.Hathwar and T.N.G.Row, The Journal of Physical Chemistry A, 114, 13434(2010), https://doi.org/10.1021/jp1100413

12. R.D.Chambers, D.Holling, G.Sandford, A.S.Batsanov and J.A.K.Howard, Journal of Fluorine Chemistry, 125, 661(2004), https://doi.org/10.1016/j.jfluchem.2003.11.012

13. V.R.Hathwar, S.M.Roopan, R.Subashini, F.N.Khan and T.N.G.Row, Journal of Chemical Sciences (Bangalore,India), 122, 677(2010), https://doi.org/10.1007/s12039-010-0056-1

14. M.B.Stringer, J.H.Bowie, J.M.Gulbis and E.R.T.Tiekink, Zeitschrift für Kristallographie Crystalline Materials, 209, 189(1994), https://doi.org/10.1524/zkri.1994.209.2.189

15. R.Subashini, F.N.Khan, R.Kumar, V.R.Hathwar and S.W.Ng, ActaCrystallographica Section E, 65 , o2721(2009), https://doi.org/10.1107/S1600536809040823

16. R.Subashini, F.N.Khan, M.Gund, V.R.Hathwar and S.W.Ng, ActaCrystallographica Section E, 65, o2723(2009), https://doi.org/10.1107/S1600536809040847

17. F.N.Khan, R.Subashini, R.Kumar, V.R.Hathwar and S.W.Ng, ActaCrystallographica Section E, 65 , o2710(2009), https://doi.org/10.1107/S1600536809040665 
RASĀYAN J. Chem.

47-63| Special Issue | 2021

18. R.Subashini, F.N.Khan, M.Gund, V.R.Hathwar and S.W.Ng, ActaCrystallographica Section E, 65, o2720(2009), https://doi.org/10.1107/S1600536809040835

19. F.N.Khan, R.Subashini, A.K.Kushwaha, V.R.Hathwar and S.W.Ng, ActaCrystallographica Section $E, \mathbf{6 5}$, o2709(2009), https://doi.org/10.1107/S1600536809040860

20. F.N.Khan, R.Subashini, S.M.Roopan, V.R.Hathwar and S.W.Ng, ActaCrystallographica Section E, 65, o2686(2009), https://doi.org/10.1107/S1600536809040653

21. S.Ladraa, A.Bouraiou, S.Bouacida, T.Roisnel and A.Belfaitah, Acta Crystallographica Section E, 66, o693(2010), https://doi.org/10.1107/S1600536810006501

22. D.E.Lynch and I.McClenaghan, ActaCrystallographica Section E, 57, o54(2001), https://doi.org/10.1107/S1600536800019590

23. R.K.Somvanshi, R.Subashini, V.Dhanasekaran, G.Arulprakash, S.N.Das and S.Dey, Journal of Chemical Crystallography, 38, 381(2008), https://doi.org/10.1007/s10870-007-9305-7

24. R.Subashini, F.N.Khan, T.R.Reddy, V.R.Hathwar and M.Akkurt, ActaCrystallographica Section E, 66, o1535(2010), https://doi.org/10.1107/S1600536810020386

25. A.D.Skolyapova, G.A.Selivanova, E.V.Tretyakov, T.F.Bogdanova, L.N.Shchegoleva, I.Yu. Bagryanskaya, L.Yu.Gurskaya and V.D.Shteingarts, Tetrahedron, 73, 1219(2017), https://doi.org/10.1016/j.tet.2017.01.026

26. R.Kimmel, M.Necas and R.Vicha, Acta Crystallographica Section E: Structure Reports Online, 66, o1261(2010), https://doi.org/10.1107/S160053681001576X

27. S.M.Roopan, F.N.Khan, R.Kumar, V.R.Hathwar and M.Akkurt, Acta Crystallographica Section E: Structure Reports Online, 66, o1543(2010), https://doi.org/10.1107/S1600536810020490

28. F.N.Khan, S.M.Roopan, A.K.Kushwaha, V.R.Hathwar, M.Akkurt, Acta Crystallographica Section E: Structure Reports Online, E66, o1544(2010), https://doi.org/10.1107/S1600536810020507

29. G.H.Stout and L.H.Jensen, Crystal Structure Determination, Macmillan Co., New York, (1968).

30. F. H. Allen O.Kennard, D.G.Watson, L.Brammer, A.G.Orpen and R.Taylor,Journal of Chemical Society, Perkin Transactions 2, 12, S1(1987), https://doi.org/10.1039/P298700000S1

31. J.E.Davies and A.D.Bond,ActaCrystallographica Section E, E57, 0947(2001), https://doi.org/10.1107/S1600536801014891

32. http://www.way2drug.com/PASSOnline/index.php.

33. D.Filimonov, V.Poroikov, Yu.Borodina and T.Gloriozova, Journal of Chemical Information and Computer Science, 39, 666(1999), https://doi.org/10.1021/ci9803350

34. V. Poroikov, D. Akimov, E. Shabelnikova and D. Filimonov, SAR and QSAR in Environmental Research, 12, 327(2001), https://doi.org/10.1080/10629360108033242

35. S.Anzali, G.Barnickel, B.Cezanne, M.Krug, D.Filimonov and V.Poroikov, Journal of Medical Chemistry, 44, 2432(2001), https://doi.org/10.1021/jm0010670

36. E.Arunan, G. R.Desiraju, R. A.Klein, J.Sadlej, S.Scheiner, I.Alkorta, D. C.Clary, R. H.Crabtree, J. J.Dannenberg, P.Hobza, H. G.Kjaergaard, A. C.Legon, B.Mennucci, and D. J.Nesbitt, Pure and Applied Chemistry, 83, 1619(2011), https://doi.org/10.1351/PAC-REP-10-01-01

37. S.Subramanian and M.J.Zaworotko, Coordination Chemistry Reviews, 137, 357(1994), https://doi.org/10.1016/0010-8545(94)03008-E

38. C. F.Macrae, I.Sovago, S. J.Cottrell, P. T. A.Galek, P.McCabe, E.Pidcock, M.Platings, G. P.Shields, J. S.Stevens, M.Towler and P. A.Wood, Journal of Applied Crystallography, 53, 226(2020), https://doi.org/10.1107/S1600576719014092

39. G.R.Desiraju and T.Steiner, The Weak Hydrogen Bond in Structural Chemistry and Biology, OUP, Chichester, (1999), https://doi.org/10.1093/acprof:oso/9780198509707.001.0001

40. P.T.Galek, L.Fábián and F.H.Allen, Acta Crystallographica Section B, B66, 237(2010), https://doi.org/10.1107/S0108768110003988

41. G. A.Jeffrey, An Introduction to Hydrogen Bonding, Oxford University Press, Oxford, (1997).

[RJC-6620/2021] 